\title{
Erratum: $R$-matrix analysis and prediction of low-energy neutron-induced fission cross sections for a range of Pu isotopes [Phys. Rev. C 88, 054612 (2013)]
}

\author{
O. Bouland, J. E. Lynn, and P. Talou
}

(Received 20 December 2019; accepted 4 March 2020; published 17 March 2020)

DOI: 10.1103/PhysRevC.101.039901

A graphic substitution has occurred in the published article regarding Fig. 18. The related caption refers to the ${ }^{239} \mathrm{Pu}$ capture cross section, whereas the graphic shows the ${ }^{241} \mathrm{Pu}$ capture cross section. To correct this unfortunate exchange, Fig. 1 in the present Erratum provides the relevant Pu isotope cross-section calculation with comparison to recent evaluated nuclear data and the old measurement by Hopkins and Diven [5] as discussed in the published article. There is also a misprint on p. 15 of the published article [item (i)] regarding the ${ }^{236} \mathrm{Pu}$ neutron-induced fission cross section which barrier heights determination has been made from the analysis of the ${ }^{237} \mathrm{~Np}\left({ }^{3} \mathrm{He}, t f\right)$ direct-reaction measurement by Gavron et al. [6] and not, as printed, from the ${ }^{237} \mathrm{~Np}\left({ }^{3} \mathrm{He}, d f\right)$ reaction.

O.B. acknowledges the anonymous referee of a recent paper [7] for the encouragement to publish this Erratum.



FIG. $1 .{ }^{239} \mathrm{Pu}$ average capture cross section computed with the AVXSF code as a function of incident neutron energy and compared to some evaluated nuclear data (ENDF/B-VIII.0 [1], JEFF-3.3 [2], JENDL-4.0 [3], and TENDL-2017 [4]) and the old measurement (black open circles) by Hopkins and Diven [5].

[1] D. A. Brown, M. B. Chadwick, R. Capote, C. Kahler, A. Trkov, M. W. Herman, A. A. Sonzogni, Y. Danon, A. D. Carlson, M. Dunn et al., Nuclear Data Sheets 148, 1 (2018).

[2] O. Cabellos, F. Alvarez-Velarde, M. Angelone, C. J. Diez, J. Dyrda, L. Fiorito, U. Fischer, M. Fleming, W. Haeck, I. Hill et al., EPJ Web Conf. 146, 06004 (2017).

[3] K. Shibata, O. Iwamato, T. Nakagawa, N. Iwamoto, A. Ichihara, S. Kunieda, S. Chiba, K. Furutaka, N. Otuka, T. Ohsawa et al., J. Nucl. Sci. Technol. 48, 1 (2011).
[4] A. J. Koning, D. Rochman, J. C. Sublet, N. Dzysiuk, M. Fleming, and S. van der Marck, Nuclear Data Sheets 155, 1 (2019).

[5] J. C. Hopkins and B. C. Diven, Nucl. Sci. Eng. 12, 169 (1962).

[6] A. Gavron, H. C. Britt, E. Konecny, J. Weber, and J. B. Wilhelmy, Phys. Rev. C 13, 2374 (1976).

[7] O. Bouland, Reexamining fission-probability data using $\mathcal{R}$-matrix Monte Carlo simulations: Application to the $\mathrm{Pu}$ fissile isotopes over the $4-8-\mathrm{MeV}$ excitation energy range (unpublished). 